\title{
De los grupos espontáneos a las comunidades de base. Fe y política en la contestación postconciliar italiana (1965-1969)
}

From spontaneous groups to Ecclesial Base Communities. Faith and Politics in the Italian Catholic Protest (1965-1968)

\author{
Alessandro SANTAGATA \\ Università di Roma Tor Vergata \\ santaga.alessandro@gmail.com
}

\begin{abstract}
The main goal of the paper is to investigate the relationship between the religious and political dimension in the Italian reception of Vatican II from the end of the Council to the explosion of May 1968. More specifically, the paper deals with the development of the Catholic protest against the Hierarchy and the political system, the development of spontaneous groups and their evolution into the Ecclesial Base Communities.
\end{abstract}

Keywords: May 68; „V Vatican Council; Italy; Base Communities; religious protest.
Resumen: El principal objetivo de este artículo es investigar la relación entre la dimensión religiosa y la política de la recepción en Italia del Concilio Vaticano II desde el final de la reunión conciliar hasta la eclosión de Mayo del 68. De un modo más específico, esta contribución aborda el desarrollo de las protestas del mundo católico contra la jerarquía y contra el sistema político, e igualmente, el desarrollo de los grupos espontáneos y su evolución en las comunidades de base.

Palabras clave: Mayo del 68; Concilio Vaticano II; Italia; comunidades de base protesta religiosa.

El objeto de este estudio es la naturaleza «política» de la «contestación católica» en Italia: un campo de investigación aparentemente fácil de delimitar, pero que necesita sin embargo de una precisión preliminar ${ }^{1}$. No hay duda de que el llamado gauchisme chrétien (tomando esta definición de la historiografía francesa) ${ }^{2}$

1 El presente artículo representa la reelaboración en forma más extensa de la conferencia dictada en el congreso «La rivoluzione del Concilio. La contestazione cattolica degli anni Sessanta e Settanta», Turín, 17-18 de noviembre de 2016. Las actas del congreso han sido publicadas con el título de Silvia INAUDI y Marta MARGOTTI (eds.), La rivoluzione del Concilio. La contestazione cattolica negli anni: sessanta e settanta, Studium, Roma, 2017, pp. 53-69.

2 Cfr. Denis Pelletier, La crise catholique. Religion, société, politique, Paris, 2002; Denis Pelletier y Jean-Louis SCHLEGEL (eds.), À la gauche du Christ. Les chrétiens de gauche en France de 1945 à nos jours, Paris, 2012. 
ha sido un fenómeno político internacional que ha alcanzado su fase de máximo desarrollo en los años sesenta y setenta (para constatarlo puede verse el cuadro ofrecido por Gerd-Rainer Horn) ${ }^{3}$. De igual manera, no se puede negar que el llamado «dissenso» (categoría italiana que debe manejarse con cuidado) ${ }^{4}$ ha sido también, y sobre todo, un fenómeno religioso e intraeclesial que se explica a la luz de la transformación del catolicismo después del Concilio Vaticano II. En una publicación reciente he propuesto como hipótesis interpretativa que la explosión de la contestación se debe colocar en el entrecruzarse de las tensiones internas del «mundo católico» italiano sobre cómo poner en práctica la enseñanza del Concilio, y la transformación de la sociedad en los años 60 en fase de galopante secularización ${ }^{5}$. En el «momento 68», y por tanto en la contaminación con el imaginario del movimiento estudiantil, las instancias de reforma solicitadas por el Vaticano II no han desaparecido, pero -como escribía ya Guido Verrucci ${ }^{6}$ - sí se han modificado, radicalizadas y enfrentadas con los vértices de la Iglesia y de sus estructuras.

Para entender esta doble naturaleza es esencial adoptar una perspectiva cronológica que tenga en cuenta la historia de las relaciones entre la Iglesia y el poder político en el siglo XX, el carácter fundamental de compromiso constitucional y los fermentos políticos y culturales madurados a partir de los años cuarenta ${ }^{7}$. $\mathrm{Al}$ mismo tiempo, el estallido de la contestación ha sido la respuesta a toda una serie de elementos que se encuadran en la cronología breve de los pocos años que separan el desarrollo del Vaticano II de los primeros y numerosos episodios de protesta. Se trata, por tanto, de una perspectiva parcial -que descuida muchas de las implicaciones de la protesta intra-eclesial (los choques sobre la reforma institucional, la doctrina, la moralidad, la libertad teológica, el ecumenismo y otros temas conectados con la esfera política en un sentido amplio)- que permiten entrar en lo específico de la «politicidad» de la contestación religiosa en sus inicios, insertando el fenómeno en su contexto eclesial y civil. Después de haber ilustrado rápidamente los puntos más relevantes respecto a la primera recepción política

3 Gerd-Rainer HORN, The Spirit of Vatican II. Western European Progressive Catholicism in the Long Sixties, Oxford, 2015.

4 Cfr. Daniela SAResella, $I l$ «dissenso» cattolico, en Marco IMPAGLIAZZO (eds.), La nazione cattolica. Chiesa e società in Italia dal 1958 a oggi, Milano, 2004, pp. 265-290.

5 La contestazione cattolica. Movimenti, cultura e politica dal Vaticano II al '68, Roma, 2016.

6 Cfr. Guido VerucCI, Il dissenso cattolico in Italia, en Studi Storici, 1 (2002), pp. 221-239.

7 Para una visión atenta al largo período cfr. Giovanni MiCCOLI, Chiesa e società in Italia dal Concilio Vaticano I (1870) al pontificato di Giovanni XXIII, en Storia d'Italia, vol. V/2, Torino, 1973, pp. 1493 1548. 
del Vaticano II (1965-1968), nos concentraremos en el tema de la ambigüedad en la retórica de aquello que eran llamados «grupos espontáneos» y de la neonata red de las comunidades de base en su postura frente a la relación entre inspiración religiosa, pertenencia eclesial y compromiso con la izquierda.

\section{LA NATURALEZA POLÍTICA DE LA RECEPCIÓN CONCILIAR}

En primer lugar es útil mostrar como el problema de las expectativas y de la recepción del evento conciliar resulta central para comprender lo que llamo su «recepción política», entendida como recepción por parte de los autores comprometidos en política y como recepción del mensaje político conciliar ${ }^{8}$. Sintetizando al máximo, el Vaticano II había ratificado la separación de la esfera de influencia de la Iglesia de aquella política con el reconocimiento de la legítima pluralidad de las opciones temporales de los católicos cuando actuaban en política «en nombre propio y como ciudadanos guiados por la propia conciencia cristiana» (Gaudium et Spes, 76). En la declaración Dignitatis humanae se encontraban asumidos los valores de la laicidad de las instituciones políticas y del pluralismo religioso. Difícilmente, este resultado y los contenidos del cambio podían ser interpretados unánimemente. Conviene tener presente que a causa de la naturaleza de compromiso, el corpus conciliar presentaba elementos de continuidad con los últimos dos siglos de «doctrina de la cristiandad» de matriz medieval. De otra parte, aún en su versión conciliadora con la democracia, la filosofía de la «nueva cristiandad» de origen maritiniano se prestaba a múltiples lecturas y planteaba algunos problemas de carácter histórico-político. En su origen, el conflicto principal no estaba tanto en los documentos -en los cuáles era posible encontrar tanto una legitimización de la «cristiandad profana», como los elementos para su arrinconamiento-, sino en la recepción de lo que era denominado el «espíritu» del Concilio.

Teniendo en cuenta estas premisas, resulta claro como la partida se ha jugado dentro del área del Concilio, en el cuál los diversos autores (institucionales y no) han leído una confirmación de las propias tesis y de la propia historia. En

8 Sobre el concepto de «recepción conciliar» cfr. Gilles RoUTHIER, Il Concilio Vaticano II. Recezione ed ermeneutica, Vita e Pensiero, Milano, 2007; Christoph Theobald, La réception du Concile Vatican II. Accéder à la source, Paris, 2009. Sobre el Concilio «como evento» veáse Giuseppe ALBERIGO, Fedeltà e creatività nella ricezione del Concilio Vaticano II, en ID., Transizione epocale. Studi sul Concilio Vaticano II, Bologna, 2009, pp. 47-69. 
una galaxia compleja de posiciones es posible identificar dos lecturas diversas del Vaticano II, pero sobre todo de la realidad italiana y de la función histórica que los católicos han asumido en ese contexto: de un lado, la línea de Pablo VI y de los vértices de las organizaciones católicas que sostenían la necesidad de integrar el Vaticano II en el proyecto de una «nueva cristiandad». Por otra parte, la línea de algunos grupos intelectuales y del catolicismo «de base», para los cuales la postura de Maritain no solo había sido sustancialmente traicionada en Italia, sino que había sido superada por la «diáspora» de los católicos entre los varios partidos y de la separación neta entre pertenencia religiosa e identidad política o al menos de su redefinición; un sentimiento común en la sociedad italiana y, de diferentes formas dentro del mundo católico en el cual la contestación de la clase dirigente ha precedido el $68 \mathrm{y}$, de alguna manera, ha puesto las bases teóricas del paso sucesivo a la contestación abierta ${ }^{9}$.

Aunque sería interesante profundizar, se dejaran de lado las divisiones internas surgidas en el episcopado, por un lado en la fase de control de la recepción conciliar en los ambientes intelectuales y teológicos (ténganse en mente episodios como la revocación del imprimatur a la revista florentina Testimonianze y la dimisión forzadas del católico de izquierdas Raniero La Valle de la dirección del cotidiano nacional católico L'Avvenire d'Italia), por otro lado en la elaboración de la declaración para las elecciones políticas del 1968, en la cual los obispos, apelando a la enseñanza del concilio sobre la defensa de la libertad de la Iglesia, habrían reafirmado su apoyo a la Democrazia Cristiana y al gobierno de centroizquierda de Aldo Moro (con los socialistas) contra el peligro comunista ${ }^{10}$. Es conveniente poner nuestra atención sobre los problemas suscitados por la recepción política del Vaticano II en las principales organizaciones del laicado. Para este fin se pueden señalar tres cuestiones cuya discusión afectaba transversalmente al mundo católico: la injerencia de la Santa Sede y de la Conferencia episcopal italiana en política (son paradigmáticos a este respecto el problema del divorcio y de la revisión del Concordato); la unidad política de los católicos en la Democracia cristiana; y la eclesiología entendida como organización de los creyentes en relación a su obediencia a la jerarquía y en relación al empeño en la sociedad. Para agravar la crisis se sumaba la creciente desafección de las nuevas generaciones a las organizaciones de la Iglesia, su acercamiento a las posiciones de izquierdas y por

9 Sobre la impronta de Maritain en la historia politica italiana, cfr. Pietro SCOPPOLA, La «nuova cristianità» perduta, Roma, 1985.

10 I cristiani e la vita pubblica (16 de enero de 1968), en Enchiridion Cei. Decreti, dichiarazioni, documenti pastorali per la Chiesa italiana (1954-1972), Bologna, 1985, pp. 481-490. 
tanto la disminución de las inscripciones a la Acción católica y a la participación parroquial. En este contexto, la fórmula de la «elección religiosa» fue la solución con la cual la cúpula compuesta por el presidente de la ACI, Vittorio Bachelet, y su asistente monseñor Franco Costa trataron de responder a las expectativas del Vaticano II; una revisión profunda de las orientaciones de los años cincuenta que, sin embargo, no ha tenido como resultado una liberación definitiva de la políti$\mathrm{ca}^{11}$. Justamente, sobre este punto se centró el choque con los sectores juveniles de la Acción Católica y de la Federación Universitaria, que propugnaban una reforma «de la base» de la estructura con la interrupción del apoyo a las iniciativas del gobierno. El punto de ruptura será la reforma del Estatuto de 1969 que favorecerá las deserciones en la asociación ${ }^{12}$. El mismo año, el Congreso nacional de la Acli (la principal organización de los trabajadores católicos) sancionaba la ruptura del colateralismo ${ }^{13}$.

$\mathrm{Al}$ igual que -teóricamente- en la ACI, los dirigentes de la Acli han puesto en el centro de la cuestión el problema de las relaciones con el partido político, aunque con resultados muy diversos. Desde su punto de vista, la acción temporal recomendada por la Gaudium et Spes sólo podía desarrollarse en estrecho contacto con los trabajadores y por lo tanto en su dimensión política y sindical. En otras palabras, el Vaticano II proporcionado la justificación para la ruptura con la Democracia Cristiana reconociendo la autonomía de los laicos en la política ${ }^{14}$. En la idea del presidente Labor, la Acli debía transformarse del movimiento de trabajadores cristianos a un movimiento de trabajadores cristianos actuando en el plano político de izquierdas sin implicar a la Iglesia en el juego que pretendían jugar en la izquierda. Aunque, si nos atenemos a los hechos, el paso resultará mucho más complicado, es importante repetir la idea de cómo el corpus conciliar ha sido interpretado y forzado para sostener lecturas y opciones políticas opuestas: si tenga en cuenta, como último ejemplo, el congreso de Lucca, organizado por la Democracia Cristiana en abril de 1967 para oponerse a las tendencias centrífugas en nombre de aquella tradición popular que, en la idea de un intelectual como Sergio Cotta y del secretario Mariano Rumor, habría sido ratificada por la Gau-

11 Para un cuadro de conjunto, cfr. Mario CASELLA, L'azione cattolica nell'Italia contemporanea (19191969), Roma, 1992.

12 Cfr. Mario CASELLA, Gli Statuti generali dell'Azione cattolica italiana (1923-1969), en Ernesto PREZIOSI (eds.), Gli Statuti dell'Azione cattolica italiana, Roma, 2003, pp. 15-95.

13 Entendido como el apoyo dado a un partido político por parte de organizaciones obreras, culturales, sociales, no integradas orgánicamente en dicho partido.

14 Para trazar un marco del asunto, cfr. Maria Cristina SERMANnI, Le ACLI alla prova della politica (1961-1972), Napoli, 1986. 
dium et Spes ${ }^{15}$. En el mismo período en que las asociaciones y el partido afrontaban esta revisión (en la continuidad), en la sociedad se iban desarrollando nuevos movimientos y diferentes formas de vida comunitaria. No se pretende ahora aquí recorrer y analizar en sus diversos aspectos la explosión de la contestación (de los contra-cuaresmales a la ocupación de la catedral de Parma, a la disputa sobre la Humanae vitae, al caso de la comunidad del Isolotto de Florencia guiada por don Enzo Mazzi $\left.{ }^{16}\right)$. Más específicamente, se examinará el discurso público de los grupos en su rápida evolución en las nuevas formas de vida comunitaria, mostrando como en la contaminación con el imaginario de la protesta global también la pertenencia religiosa y la recepción del Vaticano II se han visto complicadas en relación al problema de la identidad política del «dissenso».

\section{LA PROTESTA DE LOS GRUPOS ESPONTÁNEOS}

En su ensayo de 1983, considerado todavía ahora como uno de los más importantes sobre el fenómeno del «dissenso católico», Mario Cuminetti hablaba de la proliferación de los «grupos espontáneos» como la «señal más vistosa» del malestar creado por la gestión moderada del Concilio y del progresivo trasvase de algunos sectores católicos del empeño en la Iglesia al empeño en la políti$\mathrm{Ca}^{17}$. Esta segunda afirmación parece que puede ser sometida a revisión al menos parcialmente a la luz de un fenómeno en el cual fe y política aparecen desde los inicios como dos elementos estrechamente conectados. El primer rastro de los grupos aparece en la mitad de los años sesenta. Una investigación sobre este fenómeno, llevada a cabo por Franco Ferraresi y Franco Rositi para la editorial

15 He reconstruido el debate sobre el Concilio en la Democracia Cristiana en «I cattolici italiani nei tempi nuovi della cristianità». La DC e la ricezione politica del Vaticano II: alle origini del Sessantotto cattolico, en Mondo contemporaneo, 3 (2013), pp. 39-71.

16 Un eficaz visión de conjunto en la reconstrucción periodística de Roberto BERETTA, Cantavamo Dio è morto. Il '68 dei cattolici, Casale Monferrato (AL), 2008.

17 Mario CuminetTi, Il dissenso cattolico in Italia, Milano, 1983, pp. 125-129. Su posición estaba avalada por las observaciones de Carlo Falconi, periodista y observador atento del mundo católico, que había visto en los grupos la antecamara de la contestación del mayo del 68, y también por algunas investigaciones realizadas en el bienio 1969-1970 por Marco Boato, don Arnaldo Nesti, don Vittorino Merinas y Nando Fabro: Carlo FalCONI, La contestazione nella Chiesa, Milano, 1969; Marco BOATO, Contro la Chiesa di classe, Venezia, 1969; Vittorino MerINAS, Dossier sulla contestazione, Torino, 1969; Arnaldo Nesti, L'Altra Chiesa in Italia, Milano, 1970; Nando FABRO, I cattolici e la contestazione in Italia, Fossano, 1970. Veáse también, Giovanni BIANCHI, L'Italia del dissenso, Brescia, 1968; Ernesto ROTELLI, I gruppi spontanei del '68, en Lorenzo BEDESCHI, Luigi ACCATTOLI (eds.), I cristiani nella sinistra. Dalla Resistenza a oggi, Roma, 1976, pp. 184-198. 
Comunitá della Fondazione Olivetti, señalaba que a finales de 1968 había unos trescientos activos, localizados preferentemente en las ciudades del triángulo industrial, pero sobre todo en Emilia Romana y la Toscana. Cada uno de ellos tenía una media de diecisiete afiliados, por lo general, jóvenes, varones, católicos, instruidos y se dividían esos grupos por áreas de interés. La mayoría de ellos eran de inspiración católica (44\%) y provenían de los antiguos miembros de la Democracia Cristiana y de las estructuras del asociacionismo católico, aunque no faltaban círculos «mixtos» de católicos y no creyentes y grupos de inspiración comunista y socialista. Según los autores de la investigación la composición de los grupos dependía de la crisis de los canales institucionales de participación política, social y religiosa, y, al mismo tiempo, del deseo de encontrar un nuevo modo de «hacer política». Desde el punto de vista ideológico, la investigación indicaba que más de la mitad de los grupos se identificaban con la «izquierda cristiana» y miraban con interés a la «nueva izquierda ${ }^{18}$. Nos ayudará a entender más un análisis de cerca del nacimiento del network nacional.

Entre las primeras iniciativas del movimiento figura el congreso de Rimini del noviembre de 1967 sobre La fine dell'unità politica dei cattolici, la socialdemocrazia al potere e le prospettive politiche della sinistra italiana. El encuentro había sido preparado por el círculo local J. Maritain, guiado por Antonio Zavoli, uno de los círculos más numerosos y activos de la región, con la participación de los relatores Luigi Anderlini, del Movimiento de los socialistas autónomos, Wladimiro Dorigo, director de la revista Questitalia, Achille Occhetto, de la Federación juvenil comunista y Franco Boiardi, del Partido socialista de unidad proletaria. La discusión trataba sobre «las primeras líneas de un trabajo político nuevo para las fuerzas de la izquierda italiana, tanto a nivel ideológico, como operativo»y habría concluido con el rechazo de parte de la mayoría del apoyo a la propuesta de Lorenzo Bedeschi sobre la oportunidad de avanzar alguna candidatura católica en el Senado dentro de las listas del Partico comunista ${ }^{19}$. La primera verdadera

18 Franco FERRARESI y Franco ROSITI et al., La politica dei gruppi. Aspetti dell'associazionismo politico di base in Italia dal 1967 al 1969, Milano, 1970.

19 Para una reconstrucción del debate en las revistas católicas, cfr. Daniela SARESELLA, Dal Concilio alla contestazione. Riviste cattoliche negli anni del cambiamento 1958-1968, Brescia, 2005, pp. 416438. Una panoramica sobre los trabajos del congreso en I gruppi spontanei e il ruolo politico della contestazione, Milano, 1969, p. 5; Convegno nazionale dei «gruppi spontanei» sull'unità della sinistra, en Adista, 3 (30 de noviembre de 1967). Bedeschi ha sido uno de los interpretes de la época postconciliar en Cattolici e comunisti, Milano, 1974. Según Gianluigi Degli Esposti, que había seguido los trabajos como corresponsal de la revista boloñesa Il Mulino, el rechazo a las candidaturas había sido una importante demostración de madurez (Cattolici del dissenso o cattolici dell'attesa?, 
aparición pública de la red de los grupos haría su aparición en un contexto contra eclesial, o sea fuera de los muros de la Domus Mariae, donde se estaba celebrando la Asamblea de la Cei de febrero de 1968, con gran proliferación de pancartas alusivas al Concilio y al fin del voto confesional ${ }^{20}$. El siguiente paso será la convocatoria de un segundo congreso titulado Credenti e non credenti per una nuova sinistra (Bolonia, 25 de febrero de 1968), que supuso el bautismo de los grupos como movimientos orgánicos con la presencia de casi 600 participantes (2000 según Questitalia) representando a más de 80 círculos.

La coordinación estaba en las manos del grupo de Questitalia y por lo tanto no fue casual que la sesión de apertura estuviera a cargo de Dorigo ${ }^{21}$, el cual empezaba su intervención invitando a los grupos a que cooperaran para la superación superar la unidad política de los católicos, a participar en la fundación de una «nueva izquierda» y a distanciarse de cualquier forma de «integrismo», de derechas o de izquierdas ${ }^{22}$. La polémica era uno de los caballos de batalla del grupo veneciano que la esgrimía tanto contra el magisterio de Pablo VI y su gestión del momento post-conciliar, como contra el PCI, culpable de buscar la colaboración con la Santa Sede y el bloque católico ${ }^{23}$. En el estatuto de la $A s$ semblea dei gruppi di impegno politico e culturale per la nuova sinistra (aprobado en

en Il Mulino, 12 (diciembre de 1967, pp. 1059-1063). La revista florentina Politica (relacionada con la izquierda democristiana) sin embargo, se mostraba más crítica con los resultados del congreso y temía el riesgo de que rompiendo el diálogo con los partidos y los grupos se les estuviera condenando a ser los «gitanos de la política» (Franco BIAGINI, Violenza e non violenza, en Politica, n. 23, diciembre de 1967 , p. 6).

20 En el primer número de la revista de los grupos espontáneos, Collegamenti, aparece el comunicado del Consejo presbiteral de la diócesis de Ravena, en el cual se tomaba partido contra la propuesta de un documento electoral. La fine di un compromesso, en Collegamenti, 1-3 (enero-marzo de 1968), pp. 24-25.

21 La función de coordinación era desarrollad también por otras dos revistas: Settegiorni, cercana a la izquierda democristiana de Carlo Donat-Cattin, y la neonata Adista, la agencia de información periodística «sobre el mundo católico sobre las realidades religiosas», creada en Roma en 1967 (con el apoyo del Pci) por Franco Leonori y Adriano Ossicini. Sobre la historia de la revista, véase su número monográfico Adista. 40 anni di informazione «altra»1967-2007, 27-28 (5 abril de 2008).

22 Una relación detallada de la intervención de Dorigo en Bologna: prima tappa dell'associazionismo spontaneo per una nuova sinistra, en Questitalia, 118-119 (enero-febrero de 1968), pp. 15-20. Sobre el desarrollo de los trabajos véase la reconstrucción de Nando FABRO, I cattolici e la contestazione..., op. cit., pp. 61-67.

23 Sobre la historia de la revista veneciana, cfr. Francesco SIDOTI, «Questitalia» e la polemica sui temi dell'organizzazione politica dei cattolici, en Sergio RISTUCCIA (eds.), Intellettuali cattolici tra riformismo e dissenso, Milano, 1975, pp. 165-219; Marcello VIGLI, «Questitalia». Una via cattolica alla laicità, en Lucia CECI, Laura DEMOFONTI (eds.), Chiesa, laicità e vita civile. Studi in onore di Guido Verucci, Roma, 2005, pp. 419-434. 
la reunión del 21 de abril), los grupos declaraban que se constituían en asamblea permanente con el objetivo de actuar con todas las fuerzas de la «contestación al sistema neocapitalista internacional»: en particular, con los trabajadores y sus sindicatos y con el creciente movimiento estudiantil contra la reforma universitaria del ministro Guir ${ }^{24}$. El binomio estudiantes-obreros era, por otra parte, la consigna de los nuevos movimientos y la asamblea de Bolonia se colocaba en un contexto de movilización. No obstante -y este es un punto fundamental-aunque el documento intentaba dejar de lado el problema del post-concilio, esta postura resultaba complicada, aunque solo fuera por la naturaleza religiosa de los grupos fácilmente rastreable por sus nombres: Maritain, Esprit, Don Milani, Papa Giovanni, Persona e comunità, pero también Nicola Pistelli, Alcide de Gasperi, Luigi Sturzo. Las fuentes vienen a confirmar este extremo.

A través del primer número del boletín nacional, Collegamenti-fundado en la reunión del 14 de enero y dirigido por el grupo Presenza de Bolonia, junto con los grupos Tralci, Leonardo de Correggio y el Centro Ricerche e Studi de Udine-, es posible hacerse una idea muy precisa de cuáles eran las acciones de los grupos. En la primera editorial los autores trataban de clarificar el concepto mismo de experiencia espontánea justificado su elección de organizarse «desde abajo» como forma de contraponerse a las estructuras autoritarias del sistema capitalista y al «falso mito» de la unidad confesional de los electores católicos ${ }^{25}$. En el mismo número de la revista venía ilustrada la actividad de los últimos meses de algunos de los grupos más activos de los cuales se ofrecía un primer censo $^{26}$. En Roma, por ejemplo, los círculos ciudadanos habían organizado un

24 El documento se publica en apéndice al volumen I gruppi spontanei..., op. cit., pp. 124-130. Sigue un elenco de 73 grupos firmantes con fecha 31 de octubre de 1968. Questitalia hacía público el comité organizativo del cual formaban parte, aparte de la revista veneciana, los siguientes círculos: Amici di Questitalia (Torino); Persona e comunità (Varese); San Bonifacio (San Bonifacio, Verona); Don Milani (La Spezia); Portico (Modena); 7. Maritain (Rimini); Persona e comunità (Lucca); Resistenza (Ancona); Lorenzo Milani (Recanati, Macerata); Esprit (Pescara); Nuova sinistra (Salerno); Basilicata (Matera); F. L. Ferrari (Messina). Sobre este punto cfr. Giambattista SCIRÈ, La democrazia alla prova. Cattolici e laici nell'Italia repubblicana degli anni cinquanta e sessanta, Roma, 2005, p. 227.

25 Editoriale, en Collegamenti, 1-3, op. cit., pp. I-II.

26 En el primer elenco figuran más de 50 siglas: Entre ellas: Impegno politico (Abbiategrasso, Milano); Resistenza (Ancona) Testimonianze Minime (Alessandria); Comunità (Asti); Società nuova (Arezzo); Gruppo giovanile borettese (Boretto, Reggio Emilia); Teatro studio città di Bologna, I Tralci, M. Sarti (Bologna); De Gasperi (Brescia); Esprit (Chieti); Il Leonardo (Correggio nell'Emilia, Reggio Emilia); Nuovi incontri (Finale Emilia, Modena); Presenza operaia, Kairos (Firenze); Persona e comunità (Gallarate, Varese); Tendenza (Imola, Bologna); Esprit (Lanciano, Chieti); Don Milani (La Spezia); Persona e comunità (Lucca); Papa Giovanni (Livorno); Francesco Luigi Ferrari (Messina); Carlo Perini, G. Puecher, Centro di cooperazione internazionale (Milano); Gruppo lavoratori cristiani (San 
ayuno por la paz en Vietnam, mientras que, en Regio Emilia, el grupo Il Risveglio había acogido la presentación del último libro de Adriana Zarri, Teologia improbabile. En Mesina, en el círculo de iniciativa política Francesco Luigi Ferra$r i$, se había celebrado un congreso sobre las perspectivas de la nueva izquierda con Dorigo y Adirano Ossicini, exponente histórico de las corrientes de los católicos comunistas. En Correggio, el círculo Leonardo había organizado una conferencia sobre la no violencia y sobre la revolución cultural china; en Sesto San Giovanni (histórica ciudad obrera) los católicos de base se habían reunido para discutir la presencia de sacerdotes en las fábricas. Como se puede ver, se trata de un conjunto de iniciativas tanto políticas como eclesiales. La reforma de la Iglesia y el Vaticano II, que Dorigo había querido dejar fuera, en realidad entraban por la ventana, en particular en la polémica sobre la «restaurazione aggiornata» de Pablo VI.

En las discusiones prevalecían los grandes temas de la guerra, el neocolonialismo y el confesionalismo estatal, pero también estaban presentes los problemas de la Iglesia y las mismas cuestiones políticas eran examinadas por los grupos a la luz de las palabras del Concilio. Sobre la guerra estadounidense en Indochina, por ejemplo, el círculo de Zavoli había escrito en enero de 1967 una carta abierta a Pablo VI contra la llamada a la «guerra santa» en Vietnam invocada por el cardenal americano Francis Spellman ${ }^{27}$, carta en la cual recordaba al pontífice que después del Vaticano II y del magisterio del papa Juan, los creyentes no estaban ya dispuestos a aceptar un retorno a las cruzadas. Igualmente, en la toma de posición contra la llamada electoral de la Conferencia Episcopal Italiana, el circulo Dialoghi de Ravena denunciaba como el documento era «una vez más abstracto y privado del sentido de concreción que el Concilio se había esforzado tanto en introducir ${ }^{28}$. Al Vaticano II se había referido también el círculo Mounier de Verona en su Lettera aperta ai vescovi sulle compromissioni del

Donato Milanese, Milano); Il Portico (Modena); N. Pistelli (Palermo); Esprit (Pescara); Il Gotico (Piacenza); Fermento nuovo (Piangipane di Ravenna); Cenacolo (Salerno); I provinciali (Sarzana, La Spezia); Gruppo di ricerca (Palmi, Reggio Calabria); Nuovo impegno (Pisa); Centro di documentazione, Studi sociali, Cineforum (Pistoia); Dialoghi (Ravenna); Il Risveglio, Alternative (Reggio Emilia); facques Maritain, Astrolabio (Rimini); Centro studi sui problemi dell'uomo e della società di oggi, Pantheon, Ozanam, Ora sesta (Roma); Centro di documentazione (Torino); Persone e comunità (Varese), Centro S. Bonifacio (Verona).

27 El texto de la carta está reproducido integralmente en Marco BOATO, Contro la Chiesa..., op. cit., pp. 85-87. El 25 de diciembre de 1967 el grupo había enviado una carta abierta al papa después de su encuentro, dos días antes, con el presidente americano Lyndon Johnson (Lettera aperta al papa sull'incontro con il presidente fohnson, en Contro la Chiesa..., op. cit., pp. 103-104).

28 La carta está reproducida íntegramente en Il tetto, 25 (febrero de 1968), pp. 3-6. 
magistero gerarchico in materia di politica ${ }^{29}$, y nuevamente el Maritain, en un estudio sobre la libertad sexual del cristiano y el control de los nacimientos después de Gaudium et $S_{p e s^{30}}$. Estas iniciativas se enmarcaban en un clima de abierta y extendida contestación religiosa, iniciada simbólicamente con la ocupación por parte de los estudiantes de la Universidad Católica de Milán el 17 de noviembre de $1967^{31}$. No llama por lo tanto la atención que en el número de mayo de Collegamenti se dedicase un amplio espacio al fenómeno del «dissenso», tanto estudiantil como católico.

Los primeros meses de 1968 vieron una fuerte crecida de los grupos, que doblaban el censo recogido en el primer boletín boloñes ${ }^{32}$. Se acentuaba paulatinamente el carácter político del movimiento, aunque aún permanecía la atención a las problemáticas postconciliares. A Pablo VI se le echaba en cara el hecho de haber renunciado a poner en práctica la reforma de las estructuras eclesiásticas reclamada por el Vaticano II y de mantener una actitud de connivencia con el poder. El 3 de marzo, el círculo Maritain había difundido una nueva carta abierta en la que se pedía el cierre del Observatore Romano, juzgado como políticamente comprometido por haber condenado la dimisión de la Democracia Cristiana del dirigente de la izquierda democristiana Corrado Corghi, personalidad muy cercana a los grupos. Con la represión -escribían los grupos firmantes ${ }^{33}-$ se estaba intentando «devolver al país a las condiciones del oneroso integrismo propio del periodo preconciliar, superado gracias a la adquisición de una nueva y más madura conciencia de la presencia de los cristiano en la sociedad $»^{34}$. Concluía la carta:

Sobre los temas (la paz, el auge de los nuevos pueblos, la justicia social, etc) sobre los cuales la Iglesia ha abierto, en el tiempo conciliar y post-conciliar, un diálogo fecundo con el mundo de hoy, la Democracia Cristiana no se encuentra, ligada como

29 Lettera aperta del gruppo «Mounier» ai vescovi d'Italia sulle compromissioni del magistero gerarchico in materia politica, en Marco BOATO, Contro la Chiesa..., op. cit., pp. 61-62.

30 Matrimonio, morale e regolazione delle nascite, ibid., pp. 87-97.

31 Sobre este episodio cfr. Maria BOCCI, Un problema di identità? Alle origini della contestazione studentesca all'Università Cattolica, en Marco InVERnIZI, Paolo MARTINUCCI (eds.), Dal «centrismo» al Sessantotto, Milano, 2007, pp. 142-228.

32 Se añadían a la lista otros 49 grupos, cfr. Collegamenti, 6 (mayo-junio de 1968), pp. 4-6.

33 Además del Maritain, los círculos Presenza, Il Leonardo y Alternative.

34 Decía uno de los pasajes más incendiarios de la carta: «Hoy, después del Concilio, aún es posible oir decir (con tono autoritario y de cruzada) que discutir el discutible llamamiento de los obispos a la unidad política de los católicos es, por parte de los católicos, 'anteponer su magisterio al de la Iglesia docente, es decir, a los obispos'. ¿Es posible callar leyendo en el Observatore Romano que los laicos pueden discutir libremente un documento político de la CEI mientras no se declaren católicos?». 
está con las fuerzas del capitalismo [...] En todo esto no hay ninguna «inspiración cristiana»: solo la voluntad del poder con cualquier medio y a cualquier precio. El proceso de liberación civil, política y social que en nuestro país llevan todos los hombres, grupos y fuerzas de extracción católica no será parado por estos pronunciamientos autoritarios que se colocan al nivel de los obstáculos (empalizadas) de $1948^{35}$.

En el mismo número de la revista se publicaba otro comunicado apoyado por más de 22 signaturas entre círculos y revistas, en que se denunciaba algunos hechos recientes graves y totalmente incoherentes «con el sentido de Iglesia expresado en la asamblea ecuménica»: la ya mencionada dimisión de La Valle del Avvenire d'Italia, el alejamiento del cardenal Giacomo Lercaro de su diócesis (12 de febrero), punto de referencia de las tendencias reformadoras y del diálogo con los comunistas, y las noticias de prensa, no desmentidas, acerca del alejamiento del cardenal Michele Pellegrino de Turín ${ }^{36}$. El alejamiento de Lercaro, particularmente, fue vivido como un parteaguas de los grupos. Collegamenti hacía publica una petición de clarificación a la Santa Sede ${ }^{37}$. También cabe destacar un escrito del grupo Presenza, en el cual los autores hablaban explícitamente de traición al Concilio por parte de la «iglesia oficial» e invitaban a los creyentes a distanciarse de ella:

Aquella que hoy llamamos iglesia debería haberse repudiado a sí misma: sin embargo, estamos asistiendo, en los últimos tiempos, en contraste con un movimiento positivo (aunque limitado) de los grupos, sacerdotes e iglesias locales a un retorno perentorio de la «dictadura de la iglesia oficial y del papa», temerosa de poder perder el control de una cristiandad finalmente autoconsciente [...] Se ha llegado así a diversos actos de la «iglesia oficial»: el proceso del catecismo holandés, el ahogamiento de algunas entre las voces más libres y auténticas (como Lercaro o La Valle) [...] Nosotros sentimos apremiantemente la necesidad de rechazar claramente estos falsos aspectos de la iglesia, de cortar totalmente con ellos para ir hacia la libre búsqueda de la «esposa bella, sin arruga ni mancha (Apocalipsis)» ${ }^{38}$.

Estos textos confirman como, no obstante, la «elección política» hecha por el congreso de Bolonia, los grupos no habían perdido tanto el interés por el aggiornamento conciliar como por la confianza en el magisterio romano, acusado

35 L'Osservatore romano e i casi Albani e Corghi nelle valutazioni di alcuni gruppi, en Marco BOATO, Contro la Chiesa..., op. cit., pp. 100-102.

36 Il pericolo di un'involuzione post-conciliare in un manifesto sottoscritto da numerosi gruppi, en Collegamenti, 6 (mayo de 1968), pp. 28-29.

37 A qualcuno è dispiaciuto, en Collegamenti, 1-3 (enero-marzo de 1968), pp. 31-34.

38 Collegamenti, 8 (julio de 1968), pp. 64-66. 
de haber traicionado el Concilio. Al mismo tiempo, las cartas abiertas y los comunicados contra la represión formaban parte del programa de resistencia a la restauración concebido por el grupo de Dorigo. Como trataremos de demostrar, después de las elecciones, les dos almas del movimiento, la «política» y la «religiosa», se enfrentarán abiertamente sin haber todavía resuelto la contradicción ideológica de fondo que de hecho las unía y las hacía, en algunos aspectos, intercambiables.

Sin ser exclusivos del mundo católico, los grupos eran el producto de una nueva estación cultural; una propuesta de vía de salida, no solo de las estructuras del apostolado jerárquico, sino de la misma iglesia institucional, cuya reforma era solicitada con radicalidad, aunque con el tiempo esa reforma será cada vez más considerada como improbable. Precisamente, en virtud de este carácter, los grupos representaban una minoría extraordinariamente comunicativa, una propuesta de vía de salida no solo de las estructuras del apostolado jerárquico (laceradas en su interior por el debate sobre la fe, la política y su presencia en la sociedad), sino también de la misma Iglesia. Los sucesos del 68 modificarán, radicalizándola, la postura inicial hasta hacer difícil una demarcación precisa de las áreas de pertenencia y de identidad. De todas maneras, ya solo la experiencia del primer «espontaneismo» (1967-inicios de 1968) ha significado mucha más de cuanto dicen los datos sobre la difusión del movimiento o los artículos de prensa. Se trataba de la denuncia del obstáculo representado por el sistema político italiano al Concilio, la respuesta de los movimientos a la «Iglesia fallida», la promesa de una nueva sociedad que los militantes de base leían en contraposición a una «cristiandad democrática» y todavía profundamente ligada a los esquemas tradicionales. El hecho de que la alardeada aconfesionalidad de las asambleas nacionales se peleara con una identidad en muchos casos aun fuertemente católica, representa una muestra de que la contestación surgida del encuentro entre el Concilio y la transformación de la sociedad ha ido más allá de la batalla por la laicidad para desembocar en la utopía política cristiano-revolucionaria, con toda una serie de evidentes contradicciones y herencias de la idea de cristiandad.

\section{LA ORGANIZACIÓN DE LAS COMUNIDADES DE BASE}

Llegamos así al nacimiento de la red de las comunidades de base. Estamos aún en 1968 cuando, después de sucesivos encuentros nacionales, nuevamente en Bolonia en abril y después en Módena en junio, los grupos se reencuentran «bajo las dos torres» para resolver de una vez por todas el nudo de la pertenencia. La 
asamblea reconocía que el movimiento se encontraba frente a tres posibles soluciones: la del empeño político unitario con los partidos de izquierdas en sintonía con las tendencias electorales de los militantes (Pci o el Partido Socialista Italiano de Unidad Proletaria) ${ }^{39}$; la de una acción política de izquierda autónomo con la constitución de un movimiento para presentar en las elecciones; y la búsqueda de una acción unitaria con la contestación estudiantil, rechazando cualquier participación en el sistema de la democracia formal ${ }^{40}$. Entre los que querían una colaboración con los comunistas estaba Corghi, que en Regio Emilia había organizado una asamblea nacional de los «grupos de trabajo político» para apoyar al Pci; una opción, de nuevo, rechazada por la mayoría ${ }^{41}$. Al mes siguiente del congreso de Collegamenti (13 de octubre) no obstante, se verificará una ruptura, pero sobre otra dicotomía: aquella entre «políticos» y «religiosos». En aquella ocasión se había decidido que el boletín habría prestado atención a las dos líneas creando un suplemento ad hoc. El problema de la «distinción de ámbitos entre el plano religioso y el plano político», venía afrontado por Collegamenti en un editorial de respuesta a algunos militantes que habían criticado la decisión de haber dado espacio en la publicación al controquaresimale de Trento (marzo de 1968) ${ }^{42}$. Escribía la redacción:

39 En mayo de 1968 Dorigo concedía una larga entrevista al periódico del Psiup, Il Mondo nuovo, en el cual explicaba cómo, después de Bolonia, los grupos habían perdido su identidad confesional y se decantaban exclusivamente por el juego político como organizaciones de ciudadanos comprometidos. Sobre la cuestión del voto, después de que en el curso de los trabajos asamblearios se había tratado de la posibilidad de votar con voto en blanco, Dorigo preveía que la mayoría de los militantes votarían o por el Psiup o por el Pci con el objetivo de promocionar su transformación. Wladimiro Dorigo, Cattolici e nuova sinistra, en Mondo nuovo, 19 (12 de mayo de 1968), p. 11. Citado por Daniela SARESElla, Dal Concilio alla..., op. cit., pp. 440-441.

40 Cfr. Nando FABro, I cattolici e la contestazione..., op. cit., p. 68.

41 Como refiere Collegamenti, las Assemblee di lavoro e sperimentazione politica habían nacido después de algunos encuentros en la primera mitad del año entre Corghi, Danilo Zolo, Giorgio Giovannoni y Nando Fabro. Nascono le assemblee di lavoro politico, en Collegamenti, 10 (septiembre de 1968), pp. 52-54. Para Questitalia (125-126, agosto-septiembre de 1968, pp. 115-117), a pesar de que había sido preparada por el mismo Pci, la asamblea había mostrado la madurez del movimiento y su autonomía frente a los partidos. Diverso era, sin embargo, el juicio de Nando Fabro del Gallo, el cual temía el riesgo de que el extremismo de Dorigo alejara a los grupos de las masas obreras (12, diciembre de 1968, p. 17).

42 En la ciudad universitaria se había verificado en 1966 una de las primeras ocupaciones de un Ateneo, y en el movimiento estudiantil estaba activo un fuerte componente católico representado por Paolo Sorbi y Marco Boato. En ese milieu madurará, en marzo de 1968, la decisión de llevar la protesta a la Iglesia interrumpiendo la predicación de la homilía del predicador franciscano, conocido por sus posiciones tradicionalistas. Gottardi, cercano a las posturas de los obispos reformadores, estigmatizaba la iniciativa como un acto de violencia política, extraño a la recepción del Vaticano II. Le respondía el círculo Mounier de Verona con un documento muy duro. Entre las revistas católicas los 
Rechazamos la interpretación que ve en los hechos de Trento una incorrecta intervención en un campo puramente religioso. Rechazamos, igualmente, la conclusión de un periódico católico boloñés, Il Regno, sobre estos hechos, índice muy relevante, en esta «valiente» revista, de infiltración inmovilista. Es claro, ciertamente, que cuando las autoridades eclesiásticas intervienen autoritariamente en cuestiones políticas es legítima una contestación radical [...] capaz de inventar métodos de disenso y propuestas de participación. Y esto para restar fieles a una primogenitura laica y política, si la contestación quiere coger las raíces de aquello que condena: una sociedad, una cultura, una costumbre ${ }^{43}$.

Este largo párrafo es particularmente significativo de aquella compenetración inescindible entre las dos almas que se enfrentan en la red nacional: una especie de simbiosis que no se resolverá ni siquiera después de las decisiones tomadas entre noviembre de 1968 (IV Asamblea nacional en Rímini) ${ }^{44}$ y el 26 de enero de 1969, fecha de la asamblea de Bolonia en la que tomaron vida la red de los grupos espontáneos «orientados hacia el empeño en la Iglesia» y el Bollettino di collegamento tra le comunità cristiane in Italia ${ }^{45}$.

estudiantes de Trento recibían la solidaridad de Settegiorni, Adista e Collegamenti (5, abril de 1968, pp. 1-5). Para la documentación sobre el suceso, cfr. Contro la chiesa ..., op. cit., pp. 34-40. Cfr. Roberto BereTTA, Il lungo autunno. Controstoria del Sessantotto cattolico, Milano, 1998, pp. 74-81. En el mes de septiembre se producía la ocupación de la catedral de Parma por parte del grupo Gioventù studentesca de los Protagonisti (que formaba parte del network de Collegamenti). Se trata del episodio más famoso de una protesta que cambiaba las formas de la contestación estudiantil. Para la documentación sobre el hecho, cfr. I PROTAGONISTI, Cattedrale occupata, Firenze, 1969; Contro la chiesa..., op. cit., pp. 77-83; Roberto BERETTA, Il lungo autunno..., op. cit., pp. 146-155.

43 Editoriale, en Collegamenti, 7 (junio de 1968), pp. 1-3.

44 Según las cifras de los organizadores, estaban presentes alrededor de 600 personas, representando a 68 grupos. En el documento final se ratificaba la elección del método asambleario, el rechazo de la delegación y el objetivo de forma una «nueva izquierda». Recorriendo el documento, las únicas referencias a la religión eran la declarada oposición al «autoritarismo eclesial» y la lucha contra «el connubio concordatario» entre poder político y poder religioso. Para las actas cfr. I gruppi spontanei..., op. cit., pp. 103-107. Para la crónica y el comentario a la asamblea, cfr. Assemblea nazionale dei gruppi spontanei di impegno politico per una nuova sinistra, en Collegamenti, 11 (diciembre de 1968), pp. 7-13.

45 En el curso del año 1969, a pesar de haber superado los 300 círculos inscritos no se convocaron más encuentros nacionales de la Asamblea nacional de los grupos. A esto hay que añadir que los grupos adheridos a la Assemblea di impegno politico-culturale eran solamente una minoría, la más visible, de los círculos existentes. Escribe Fabro: «en 1966 y en 1967 se hablaba de 1000 e incluso de 2000 grupos. De los datos se desprendía, en febrero de 1969, que solo una minoría de los grupos era consciente de la necesidad de un nexo de unión, en vistas a un conocimiento recíproco y a una eventual presencia y acción común [...] La mayoría de los grupos -al menos 600- parecían orientados a vivir a su aire, cada uno como un grupo de opinión. Nando FABRO, I cattolici e la contestazione..., op. cit., p. 94. Fabro se refiere al Anagrafe dei gruppi, en Collegamenti, 12 (febrero de 1969), pp. 1-11. 
No es posible entrar en el detalle de las discusiones asamblearias y de las diversas posiciones en el campo de discusión. En síntesis, se puede decir que en la red se iban delineando dos tendencias: una estaba orientada a promover una renovación interior y comunitaria, la otra retenía indispensable remover antes algunos vicios estructurales de la Iglesia que hacían imposible la renovación misma. Las dos líneas se resentían de la influencia creciente del llamado «modelo latinoamericano» de recepción conciliar elaborado en Medellín. Juzgando que las dos opciones no fueran contradictorias, las comunidades decidían no salir de la red de Collegamenti, sino de dotarse de su propia network. La redacción del Bollettino era confiada a los representantes de una decena de grupos que se proponían reencontrarse mensualmente en diversas localidades para ir reorientando la ruta. El trabajo práctico de publicación era llevado a cabo por los florentinos de Comunità e ricerca biblica. En la editorial del primer número (mayo de 1969) se podía leer el programa de coordinación nacional y un cuadro de la situación político-eclesial de referencia:

Nos parece que en el cristianismo se pueden encontrar tres presencias operativas principales, distintas entre ellas, diferenciadas, pero a menudo confluentes [...]

a) Los grupos espontáneos. Para entender lo que han sido y lo que son [...] hay que entender el deseo de libertad y de hacer cualquier cosa más allá de las coerciones institucionales que los han originado. Por otra parte, muchos ex-católicos militantes han confluido en el espontanismo. La matriz es distinta: político cultural religiosa. Después del cambio de punto de vista del año pasado, los grupos han precisado su empeño político, mientras otros se han definido como eclesiales.

b) Saltan al primer plano las verdaderas y propias comunidades cristianas: parroquiales, p. ej. El Isolotto: en territorio parroquial, p. ej. El Vandalino, inter-parroquial e inter-ciudadano, p. ej. Comunidad de Padua/Vicenza: junto a los grupos que intentan hacer o ya hacen experiencias comunitarias, p. ej., el Cenáculo de Salerno.

c) Se constituyen Movimientos a nivel ciudadano con acción de sensibilización de la opinión pública, análisis de las estructuras de las diócesis, invitación a la acción cristiana y testimoniante. En Génova el Movimiento S. Camilo, en Milán la Asamblea eclesiástica, en Roma el Centro de comunicación y la Asamblea de las iniciativas eclesiales de base.

Resumiendo, el boletín se proponía conectar estas diferentes experiencias eclesiales que consideraban unidas por un fin común: «la voluntad de llegar en nombre del Evangelio, a la auténtica liberación del 'pueblo' de todos los pesados condicionamientos, de la naturaleza que fueran, desde los económicos a los sagrados, y que le hacían imposible o muy difícil vivir como 'pueblo de Dios'». Que 
después la real composición del movimiento hiciese más difícil la acción unitaria «desde abajo» se pone en evidencia ya desde las primeras investigaciones sobre el fenómeno ${ }^{46}$.

Según los datos ofrecidos por la encuesta de Roberto Sciubba y Rossana Sciubba Pace, la composición social y cultural de las comunidades era similar a la de los grupos (clase media intelectual), pero con una mayor presencia obrera, sobre todo en las regiones meridionales ${ }^{47}$. Era común la crítica a la Iglesia institucional, acusada de estar aliada con el poder, y la aceptación del método marxista, mientras que era una novedad respecto a los grupos la presencia (en la mayoría de los casos) de sacerdotes, a veces ex sacerdotes o suspendidos por la autoridad. Nacidas de los grupos espontáneos, las comunidades de base presentaban muchas afinidades con aquella experiencia, de las que se distanciaban por su renovada inspiración religiosa y sobre todo bíblica y litúrgica. Más en general, éstas advertían la necesidad de inspirarse en el Vaticano II para refundar la comunidad cristiana que los grupos no habían dejado de lado, pero que si habían dejado fuera de la $A s$ semblea di impegno politico porque eran contrarios al uso de motivaciones religiosas para justificar las acciones políticas, aunque -como ya se ha dicho- no siempre con una praxis coherente con esta premisa.

Se dejarán ahora de lado otros aspectos de gran interés que se refieren a la relación de las comunidades con la autoridad eclesiástica, la presencia y la función del clero, y el modo de vivir los sacramentos de manera comunitaria y autogestionada, para volver directamente a la relación entre inspiración de fe y acción política $^{48}$. Como queda claro del vaciado del Bollettino de 1969-1970 y de una mirada más atenta a las experiencias tales como las del Isolotto, San Camilo de

46 Editoriale, en Bollettino di collegamento fra comunità cristiane in Italia, 1 (mayo de 1969), pp. 1-3. En el índice, colocado al final del primer número, figuraban cerca de 22 adherentes. Entre ellos: Assemblea ecclesiale e Centro San Ferdinando (Milano); Movimento «S. Camillo»; Il Cenacolo (Salerno); Centro di collegamento (Roma); las dos comunidades de Casale Monferrato referentes a don Aldo Ferrarino y a don Pier Cesare Bori; Mounier (Verona); Protagonisti (Parma), Isolotto, Peretola, Resurrezione (Florencia); Vandalino (Turín); Chiesa povera (Rímini); Centro Studi e Dialoghi (Ravena).

47 Roberto ScIUBba y Rossana ScIUBba PaCE, Le comunità di base in Italia, Roma, Coines, 1976, II, pp. 11-23.

48 Sulla storia delle comunità cfr. Roberto SCIUBBA, Rossana ScIUBBA PACE, Le comunità di base..., op. cit.; Rafael J. KleINER, Gruppi di base nella Chiesa italiana. Obiettivi e metodi di lavoro, Assisi, 1978; Massa e Meriba. Itinerari di fede nella storia delle comunità di base, Torino, 1980; Fausto PERRENCHIO, Bibbia e comunità di base in Italia. Analisi valutativa di un'esperienza ecclesiale, Roma, 1980; Massa e Meriba. Itinerari di fede nella storia delle comunità di base, Torino, 1980; Filippo GENTILONI, Marcello VIGLI (eds.) Chiesa per gli altri. Esperienze delle CDB italiane, Roma, 1985. 
Génova y la comunidad del Vandalino de Turín ${ }^{49}$, en las comunidades la necesidad de traducir la inspiración cristiana en la sociedad y, en particular, en el movimiento obrero seguía siendo muy fuerte (habla por sí solo el célebre catecismo redactado por el grupo de Mazzi que, como se lamentaba el padre De Rosa en La Civiltà Cattolica: «indicaba como héroes 'cristianos': Luther King, Camilo Torres, Malcom X, pero ningún santo de la Iglesia Católica $\gg^{50}$. Más útil para entender el framework teórico son las primeras asambleas de la red nacional. En la primera oficial, celebrada en Bolonia en septiembre de 1969, figuraba en el orden del día la superación de la jerarquía, acusada de connivencia con el capitalismo, la «desclericalización», la abolición de los dogmas y la lucha por el socialismo. Las duras críticas al episcopado dependían también de las intervenciones represivas del último período, in primis el alejamiento de Mazzi del Isolotto. Sobre política se encuentran posiciones diversas pero esta vez las intervenciones tocaron el punto central de la pertenencia religiosa: algunos aceptaban la lucha de clases, otros la rechazaban por estar poco inspirada en el punto de vista cristiano, y otros la consideraban un hecho técnico y no religioso ${ }^{51}$. Si bien la necesidad de comprometerse políticamente era aceptada por la mayoría, las posiciones se dividían entre las comunidades que se definían como socialistas, en cuanto cristianas, y aquellas que no motivaban su elección socialista en bases religiosas. La misa contraposición se constató en el congreso de octubre de 1971, considerado como el acta de bautismo del movimiento. Sin descender a los particulares, el congreso ha sido, probablemente, el principal motivo de choque entre las diversas almas de la red de las Comunidades de Base (revolucionarios en cuanto cristianos, cristianos y revolucionarios y cristianos apolíticos). El punto de encuentro estaba en el lema

49 Sobre la historia de las dos comunidades y sobre la bibliografía de referencia reenvío a mi libro La contestazione cattolica..., op. cit., pp. 236-251.

50 Giuseppe DE RosA, Il «catechismo» dell'Isolotto, en La Civiltà Cattolica, 2845 (4 enero de 1969), pp. 30-40. Sobre la experiencia de Isolotto y sobre don Mazzi contamos con una amplia bibliografía. Cfr. Christian DE VITO, Mondo operaio e cristianesimo di base. L'esperienza dell'Isolotto di Firenze, Roma, 2011. Entre los acervos de fuentes: Centro Documentazione Pistoia, L'Isolotto e il popolo di Dio. Cronologia dei fatti. Documenti, lettere su l'Isolotto e il Popolo di Dio, Milano, 1969; Comunità dell'Isolotto, Isolotto 1954-1969, Roma-Bari, 1969; Comunità dell' Isolotto, Isolotto sotto processo, Roma-Bari, 1971; Comunità dell'Isolotto, Liberarsi e liberare, Pisa, 1973. En cuanto a las memorias: Enzo MAZZI, Cristianesimo ribelle, Roma, 2008; Idem, La forza dell'esodo, Roma, 2001; Idem, Il valore dell'eresia, Roma, 2010. Un guía del archivo histórico de la comunidad en: http:// www.comunitaisolotto.org/Archivio/Archivio.htm.

51 Enzo FranchINI, La prima assemblea delle comunità ecclesiali spontanee, en Il Regno, 188 (1 de octubre de 1969), pp. 370-371. Véanse también los análisis de Arnaldo Nesti y de Enzo Bianchi referidos en Mario CUMINETTI, Il dissenso cattolico..., op. cit., pp. 191-193. 
del convenio europeo de julio de 1969 celebrado en Coira: «liberar a la Iglesia para liberar al mundo»: era como decir que sin la lucha política tampoco se daría la liberación religiosa y, sobre todo, que sin una verdadera reforma de la Iglesia -realización plena de la colegialidad, revisión profunda de la doctrina, superación definitiva de la «era constantiniana»- no era hipotizable ni siquiera la toma del poder $^{52}$. Las comunidades que participaban en la red querían, por lo tanto, «otra Iglesia» y no una «Iglesia otra».

No faltaban experiencias diversas al modelo contestatario y clasista que merecerían una profundización más específica, como la comunidad ecuménica de Bose, convertida después en un monasterio; la de San Egidio, fundada en Roma en 1968 por el estudiante Andrea Riccardi y activa en el apoyo a las formas de desarraigo social en los barrios populares de la capital; o la ya mencionada experiencia de vida comunitaria-familiar de Casale Montferrato (sustancialmente lejana de la militancia política y centrada en la lectura de la Escritura y en la vida de comunión $)^{53}$. Se movían en esta dirección, finalmente, las otras comunidades que en octubre de 1969 se encontraron en Valenza Po (Alessandria) alrededor del grupo local Il Dialogo con la participación de Pier Cesare Bori de la comunidad de Casale Monferrato, de Enzo Bianchi de Bose, y de la revista genovesa Il gallo, que hizo la crónica del encuentro ${ }^{54}$. Resultará más claro en este punto que la experiencia de las comunidades no puede ser reconducida exclusivamente al área del gauchisme y necesita comprenderse en el contexto más amplio de los movimientos post-conciliares (italianos e internacionales). Volviendo a la cuestión central de este artículo, se puede, de todas maneras, observar como en la red nacional de las Comunidades de Base a las motivaciones de los primeros grupos del movimiento se añadían nuevas consignas que provenían de la teología de la revolución y del neo-marxismo heterodoxo. Ofrece una ulterior confirmación de esto el debate sobre la cuestión de la violencia revolucionaria y su legitimidad.

Demos un pequeño paso atrás en la cronología y volvamos al agosto de 1968, fecha en la que se realiza el famoso viaje de Pablo VI a Colombia. Como

52 Fausto Perrenchio, Bibbia e comunità..., op. cit., pp. 33-34.

53 Sobre Bose cfr. Servitium, 3 (marzo 1969), pp. 679-686. Mario TORCIVIA, Il segno di Bose, Casale Monferrato, 2003. Sobre la historia de San Egidio cfr. Andrea RICCARDI, Sant'Egidio, Roma e il mondo. Colloquio con 7.-D. Durand e R. Ladous, Cinisello Balsamo, 1997. Sobre las experiencias de Casale Monferrato, cfr. Pier Cesare BorI, CV, Bologna, 2012, pp. 67-89.

54 Nando FABRO, Un incontro di gruppi a Valenza Po, en Il gallo, 12 (diciembre de 1969), pp. 1415. Otro encuentro de este tipo se desarrolló en Bose del 28 al 30 de diciembre de 1969 con Bori, Bianchi e Enzo Balducci. Una crónica en Un incontro di gruppi ecclesiali piemontesi, en Testimonian$z e, 120$ (diciembre de 1969), pp. 909-920. 
es conocido, el evento había suscitado grandes expectativas, pero también una cierta turbación de lo que da testimonio las multitud de cartas enviadas a Roma por grupos de laicos y sacerdotes sudamericanos que pedían al papa que se pronunciara sobre los problemas de la pobreza y de los problemas políticos del continente y expresaban el temor de que la visita pudiese legitimar al gobierno colombiano $^{55}$. Por lo respecta a los grupos, Collegamenti se preguntaba hacia donde iba la Iglesia en Sudamérica, desde el momento en que «a las instancias profundas del concilio y de las comunidades locales», Pablo VI había respondido «con un nuevo 'credo' superficial y dogmático y con una aparición propia de un rico en un país hambriento» ${ }^{56}$. El número de la revista presentaba una recopilación de documentos provenientes de Sudamérica y de los grupos espontáneos italianos. Entre estos últimos estaba la llamada que Corghi había dirigido a Lercaro, elegido como Legado pontificio del Congreso Eucarístico como «contrapartida» por su remoción, para que se encontrara con la madre de Camilo Torres ${ }^{57}$. De los grupos llegaba, finalmente, la petición de extender la revolución también a Italia («dentro de nuestro Viet-nam» y siguiendo el modelo de Guevara), aunque no todos compartían la elección de la violencia como instrumento preferente para la acción política. Los militantes del grupo del Centro Ricerche e Studi de Udine, por ejemplo, se disociaban de la petición de un público repudio al papa y rechazaban la adopción de la violencia como método de acción política ${ }^{58}$. A ellos les respondían los editorialistas del grupo Presenza de Bolonia, encargados de la revista:

Conviene distinguir la postura «no violenta», que debe ser una constante en el hombre, de la elección política particular que puede llevar a la guerrilla y a la revolución; y esta última elección, justamente por ser una elección política, no la avalamos con ningún apoyo doctrinal o teológico [...] La elección está clara entre capitalismo y socialismo y no admite vías intermedias (el reformismo es sustancialmente neocapitalismo) [...] No tenemos ninguna intención, al hacer estas afirmaciones, en profundizar en los datos eclesiásticos, porque es suficiente para expresar el disenso el hecho de ser hombres conscientes y razonables. Es en el plano de la acción temporal

55 Daba noticia de ello, Note di cultura, 39-40 (junio-julio de 1968), pp. 383-410.

56 Collegamenti, 8 (julio de 1968), pp. I-IV. Veáse también, Documentazione «speciale sulla Colombia», ibid., pp. 1-63.

57 Corrado CoRghI, Lettera al cardinale Giacomo Lercaro legato pontificio al Congresso eucaristico di Bogotà, ibid., p. 15. Algunos de estos documentos y cartas contra el papa han sido publicados en Contro la chiesa..., op. cit., pp. 488-496.

58 Del «Centro Ricerche e Studi» (Redazione di Udine-Sez. Documentazione), en Collegamenti, 9 (agosto de 1968), pp. I-III. 
donde disentimos y rechazamos la obra de la jerarquía. No podría ser de otro modo: hoy la jerarquía se está significando cada vez más en el plano político y temporal y cada vez menos en el plano religioso y auténticamente eclesial.

Concluía el documento sobre el problema de la relación con la Iglesia:

Ser iglesia hoy, para nosotros, no es ya un discurso que debe hacerse con citas de los textos pontificios rozando los límites de la ortodoxia (y más bueno es el que consigue ser «vanguardista» sin llegar a herético), sino una vida que debe llevarse de un modo comunitario en la única profundidad auténtica que es la de las relaciones humanas [...] Misterio en su etimología significa callar. Callar, porque es distinto el lenguaje y la presencia de Dios, del lenguaje y la presencia del hombre; y también la iglesia, para reconocer la existencia de Dios, debe ser fiel a la ley paulina, según la cual es necesario morir para que Cristo viva ${ }^{59}$.

La justificación de la violencia por parte del grupo boloñés no tenía fundamentos religiosos, sino políticos. No reconocía a la Iglesia el papel de maestra y no buscaba en la doctrina (ni siquiera en la del Concilio) una justificación de tipo religioso. La lección que llegaba del continente latinoamericano contribuía a reforzar las convicciones de quien veía en el Concilio y en la pastoral sobre la «Iglesia pobre» un mensaje de liberación no solo del pecado, sino también del sistema económico y político que por aquel entonces era objeto de una contestación cada vez más radical y amplía. El empuje revolucionario, ligado a una visión histórica de la fe, planteaba, por otro lado, el problema de la legitimidad evangélica de la violencia contra la opresión, con toda una serie de implicaciones que afectarán en los años siguientes al problema de las relaciones con la lucha armada en Italia ${ }^{60}$. Por otra parte, aunque no es posible ofrecer una estimación del fenómeno, sabemos que en muchos casos militantes provenientes de mundo católico desembarcaron en las formaciones políticas de izquierdas abandonando totalmente la militancia religiosa (Potere Operaio, Il manifesto y Lotta Continua $)^{61}$. Para aquellos que preferirán adoptar un perfil alternativo, encontrarán su lugar en Cristiani per il socialismo, movimiento nacido en Chile, en 1971, cuya sección italiana se inauguraba en el congreso de Bolonia de 1973.

59 Ibid., pp. III-VII.

60 Cfr. Guido PanvinI, Cattolici e violenza politica. L'altro album di famiglia del terrorismo italiano, Venezia, 2014.

61 Un perfil en Angelo Ventrone, Vogliamo tutto. Perché due generazioni hanno creduto nel mito della rivoluzione 1960-1988, Roma-Bari, 2012. 
El análisis de este punto nos llevaría muy lejos. Basta con decir que en la primera mitad de los años setenta las posiciones se harían cada vez más articuladas con el nacimiento de nuevas revistas que actuarían como nexos de unión como Lettere, Idoc-internazionale y Com (más tarde Com-Nuovi Tempi, nacida de la fusión con la revista valdense dirigida por Giorgio Girardet ${ }^{62}$ ) y nuevos movimientos como el 7 novembre 1971, evolución del grupo europeo de los «sacerdotes solidarios» que, después de la primera reunión en Coira, se había reunido en Roma en octubre de 1969 a la vez que se celebraba la reunión del Sínodo de Obispos ${ }^{63}$. Inspirados en la retórica de don Giulio Girardi, los Cristiani per il socialismo aun estando en primera fila en las batallas por el referéndum sobre el divorcio de 1974 y la abolición del Concordato, no se contentaron sólo con una reforma de la Democracia Cristiana o su disolución, sino que, en cuanto cristianos y militantes de izquierdas, pretendieron dirigir los creyentes hacia una lectura materialista y revolucionaria de la Biblia y hacia lugares de enfrentamiento social con la posibilidad de una confluencia de objetivos con los grupos extraparlamentarios. El tema de la reforma de la Iglesia estará siempre presente, aunque a veces quedara en un segundo plano por la desconfianza en un cambio desde arriba: en este sentido, piénsese en los efectos de la proclamación den nuevo Credo en junio de 1968 contra las vanguardias teológicas internacionales; o al schock causado por la condena de los anticonceptivos en la Humanae vitae ${ }^{64}$; o las nuevas intervenciones represivas (incluso con la ayuda de las fuerza pública como en los casos de Gioiosa Jonica y Conversano) en detrimento de personajes señalados como Girardi, Gerardo Lutte y Ivan Illich y de exponentes de algunas comunidades como don Marco Bisceglia de Lavello y don Giovanni Franzoni de la comunidad de San Pablo en Roma ${ }^{65}$. Pero sobre todo, la relación con la Iglesia institucional se hará cada vez más débil, con un discurso que alimentaba voluntariamente la ambigüedad de un movimiento que no se definía socialista en cuanto cristiano, sino que sostenía el cristianismo como un elemento revolucionario de

62 Sobre los movimientos evangélicos y la nueva izquierda, cfr. Gabriele DE CECCO, Fede e impegno politico. Un percorso nel protestantesimo italiano, Torino, 2011.

63 Cfr. Carlo Crocella, Una singolare espressione del dissenso cattolico negli anni Settanta: il movimento «7 novembre 1971», en Camillo BREZZI (eds.), Democrazia e cultura religiosa. Studi in onore di Pietro Scoppola, Bologna, 2002, 447-457.

64 Véase la carta de algunos grupos en favor de los métodos anticonceptivos (Persona e Comunità de Roma y los grupos locales de Lucca, Milano y Varese y La Spezia) y el ataque a la encíclica del grupo Presenza en Contro la chiesa..., op. cit., pp. 503-504, 117-120.

65 Cfr. La contestazione cattolica..., op. cit., pp. 221-223, 244. 
tipo socialista, llevando, de esta manera, hasta sus últimas consecuencias las contradicciones que se han intentado poner de manifiesto en esta reconstrucción de los hechos ${ }^{66}$.

\section{CONCLUSIONES}

Como se desprende de la bibliografía y de las actas de los encuentros nacionales, en los años 70 la red de las comunidades de base tratará de resolver los problemas ideológicos que hemos analizado acercándose lentamente también al mundo católico oficial ${ }^{67}$. En esta perspectiva más amplia, las experiencias de los cristianos por el socialismo y de las comunidades de base pueden considerarse como dos resultados diversos, aunque afines, de aquella fase de radicalización iniciada en el bienio 1968-1969: dos intentos de conjugar una renovada visión de la fe (y de la presencia en la Iglesia) con una nueva concepción de la militancia política que encontraba en la «nueva izquierda» la consigna para un cambio radical de la sociedad: en los dos casos, como ha sido dicho, no sin continuas laceraciones y herencias del mito de la cristiandad. ¿Cómo era posible, por otra parte, teorizar la autonomía política de la elección de las clases con la búsqueda de una «correspondencia» de esta última con un compromiso de fe? ¿Cómo se puede conjugar a la vez el amor cristiano y la tentación de la violencia revolucionaria? De este imaginario, radical, minoritario, pero fuertemente performativo, se ha tratado de mostrar cuál era su relación con la transformación en acto del catolicismo italiano. Desde este punto de vista, el concepto mismo de «política» necesita ser declinado de diversas maneras (piénsese, por ejemplo, en el debate eclesiológico entre defensores del apostolado jerárquico y los movimientos), así como han sido diversas las modalidades con las cuales los católicos han tomado parte en las contestaciones: como estudiantes, como trabajadores, como militantes; como creyentes y como ciudadanos que forman parte de un nuevo imaginario

66 Sobre la historia de Cristianos por el socialismo, cfr. Josè RAMOS REGIDOR y Aldo GECCHELIN (eds.), Cristiani per il socialismo. Storia, problematiche e prospettive, Milano, 1977; Giancarlo MILANESI, Identità religiosa e impegno politico nei «Cristiani per il socialismo», en Religione e politica. Il caso italiano, Roma, 1976, pp. 160-166.

67 Un momento importante será el IV Congreso nacional de Nápoles (23-25 de abril de 1977), en el cual el movimiento decidirá separar el elemento espiritual de la acción política. Cfr. Luigi ACCATTOLI, Le comunità di base, en Chiesa in Italia 1975-1978, Brescia, 1978, pp. 158-165; Fausto Perenchio, Bibbia e comunità..., op. cit., pp. 33-34; Mario CuminetTi, Il dissenso cattolico..., op. cit., pp. 250-261. 
generacional de carácter antiautoritario de por sí extraño a las cuestiones tratadas por el Vaticano II $^{68}$. Sin embargo, justamente en el debate-choque sobre la recepción política del Vaticano II se ha jugado una partida fundamental en el mundo católico que afectaba al carácter de fondo del sistema republicano, la presencia de los católicos en la sociedad política, la necesidad, expresada también a través de lenguaje marxista-revolucionario, de una cambio de las estructuras sociales y políticas en una fase de transformación profunda y de secularización cultural que había de hecho obligado a los católicos italianos a un papel de minoría en la sociedad. En este contexto el 68 ha representado un segundo momento de aceleración y modificación de los caracteres del enfrentamiento, generando nuevas alianzas y nuevas complicaciones en el modo de vivir la relación entre fe y política, dos elementos sustancialmente inescindibles en el análisis de la nueva izquierda católica de los años sesenta y setenta.

68 Para una visión de conjunto, cfr. Aldo AgOsti, Luisa PASSERINI y Nicola Tranfaglia (eds.), La cultura e i luoghi del '68, Milano, 1991 y la más reciente reconstrucción de Marcello FLORES y Alberto De Bernardi, Il Sessantotto, Il Mulino, 2003; Anna Bravo, A colpi di cuore. Storie del '68, Roma-Bari, 2008. 